\title{
Foreword
}

\section{A much needed new journal in the field of Pediatric Genetics}

\author{
Anne M. Slavotinek* \\ Department of Pediatrics, Division of Genetics, University of California, San Francisco, CA, USA
}

The fields of Pediatrics and Medical Genetics are closely intertwined, with specialists from both disciplines collaborating to evaluate and manage patients with diverse presentations ranging from birth defects to developmental delays of unknown cause. The need for an open forum for discussion and for manuscripts that integrate and relate to both fields has never been more pressing for the following reasons:

Firstly, newer technologies, such as array comparative genomic hybridization for testing for submicroscopic chromosome aberrations and 'next-generation' sequencing for genomic mutation detection, are reshaping the landscape of medicine. These technologies enable an astonishing amount of potentially relevant health information to be obtained from a single test, and will increasingly be employed where there are sufficient funds to sustain their use. The investigations, in particular arrays, are often performed in individuals from the pediatric age groups - for example, during the newborn period, when physical differences may be noted, and during the first few years of life, when developmental or behavioral disabilities may be recognized. When genomic testing is performed in the pediatric population, the health information that emerges from the results can have implications that extend across the future lifetime of an individual. However, the results are often more immediately important to those caring for the child. The information returned from the newer genomic tests can range from results that are expected and that confirm well-characterized diagnoses, to results that are completely unanticipated and for which the

*Address for correspondence: Anne M. Slavotinek, Department of Pediatrics, Division of Genetics, University of California, San Francisco, CA, 94143-0748, USA. E-mail: slavotia@peds.ucsf.edu. interpretation remains debatable or uncertain. We still have much to learn regarding the best methodology for the consent process and anticipatory counseling for genomic testing, in addition to the optimal follow up for families who receive information that is currently not interpretable. There is an essential need for Pediatricians and Clinical Geneticists to be familiar with these newer genetic tests, their attendant power and advantages, and the difficulties associated with their use.

Secondly, there is a trend towards the individualization of care based on genotype, in particular regarding therapeutic interventions, that has been termed 'personalized medicine'. For example, the side effects and toxicity from selected drugs can be diminished or avoided by screening for genetic variations that can alter drug metabolism. Such pharmacogenomic testing may be particularly relevant to children, in whom drug selection and dosage can be more hazardous than for adults. Pediatricians and Clinical Geneticists must become increasingly skilled in searching the literature for such advances and accurately translating the newer scientific findings into information that is appropriate for families and other specialists. They must also decide on the most efficacious 'evidence-based' management options that can directly aid patients and families. Personalized medicine focuses on the preventative aspects of healthcare, providing information that can inform lifestyle and behaviors to minimize the risks of future disease development. As the groundwork for individual health behavior is often established in childhood, pediatricians have a unique chance to positively influence future health choices by recommending proven interventions that are tailored towards the individual.

Finally, the amount of information available concerning the clinical aspects of diseases and syndromes 
and their causative genes has grown exponentially in recent years for both common and rare conditions. This information has improved the chances that an individual will be accurately diagnosed due to better descriptions of syndromes and diseases and because of an increasing wealth of genetic tests that can be obtained for specific conditions. Genetic evaluations have therefore had more to offer to families with children and to their caregivers, and an awareness of the depth and breadth of available information is vital for successful patient care. For patients from the pediatric age groups, the recurrence risks and reproductive counseling that may ensure from a confirmed diagnosis may be particularly salient for parents. The widespread use of genetic testing has lead to the discovery of many new chromosomal conditions and neurogenetic syndromes that are still being clinically characterized.
For many of these rare conditions, there is an overwhelming need to continue to document and publish the clinical presentations, testing results and current state of knowledge to provide more data for future families and their caregivers. Pediatricians must keep pace with this new information, learning the cardinal features of the more common, newly described conditions and syndromes and when genetic testing is likely to prove most valuable.

We therefore celebrate the arrival of a new journal that can provide more pediatric genetic information so that the ultimate aim of optimal patient care becomes more achievable. It is an exciting time for medicine, as novel technologies and approaches promise to transform patient care at an increasingly rapid rate. The need for accurate and timely clinical data has never been more critical. 